\title{
Slowing time cosmology with initial violetshift and three types of redshift
}

\begin{abstract}
Zahid Zakir
Abstract

In general relativity, the stretching of the wavelengths of photons in the expanding universe occurs along the path and does not depend on the velocity of the source. Therefore, the photons from the sources at rest relative to us did not have, and from the sources comoving the expansion there was an initial Doppler redshift, and then on the way both photon fluxes acquired a stretching redshift. As the result, the redshift of the comoving the expansion sources should be at least doubled. But observations show a single redshift already in the linear part, and therefore in cosmological models only with redshifts (Friedmann's and others) there was the double redshift problem with one hundred percent discrepancy between theory and observations. The observational fact of single redshifts means that the photons should have an initial violetshift, which was compensated for along the way by one of two types of redshift. In the model of slowing time cosmology (STC) proposed in 2020, the rate of proper times was higher in earlier epochs, which led to the violetshift, compensated along the way by the stretching redshift. As a result, in STC the observed shift is reduced to the initial Doppler redshift, to which the gravitational redshift is added for distant objects. The relativistic aberration then leads to dimming of the apparent luminosities. The basic relations of STC are presented, including the "distance modulus - redshift", which are consistent with observations at new values of cosmological parameters. Evolution in early epochs and its influence on the properties of CMB are also discussed. In STC the light velocity was higher in the past and for this reason it has no previously known cosmological problems.
\end{abstract}

Keywords: cosmological models, expansion of the universe, cosmological redshift, Doppler effect, gravitational redshift, dark energy, $C M B$

\section{Content}

Introduction 2

1. Two redshift mechanisms and the double redshift problem ....................................................................3

1.1. Kinematical redshift and wavelength stretching in cosmology ................................................ 3

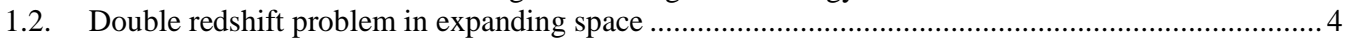

2. Two new frequency shifting mechanisms and a solution to the double shift problem .......................... 6

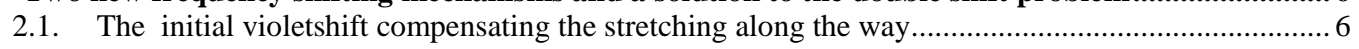

2.2. Gravitational time dilation and its contribution to redshift.................................................. 8

2.3. Observed redshifts as a result of the Doppler effect and GRC ................................................... 10

3. Slowing time cosmology (STC) taking into account the effects of gravity ...........................................11

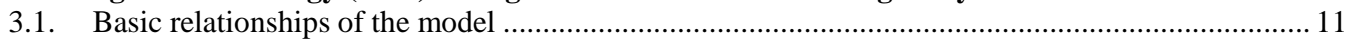

3.2. «Distance modulus - redshift» relation....................................................................................... 12

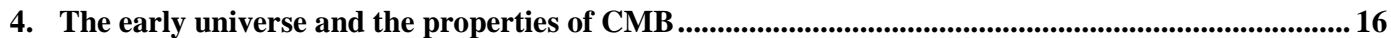

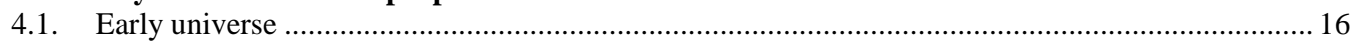

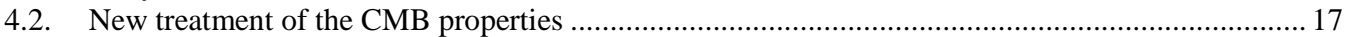

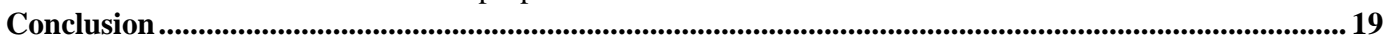

Appendix. Relativistic aberration in cosmology .............................................................................................. 19

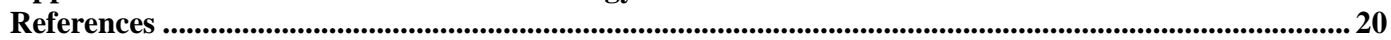

${ }^{1}$ Center for Theoretical Physics and Astrophysics, Tashkent Uzbekistan, zzakir@qgph.org, ORCID 


\title{
Introduction
}

\begin{abstract}
"It is desirable to emphasize the special necessity in the field of cosmology of avoiding the evils of autistic or wish-fulfilling thinking... The problems of cosmology are necessarily extensive and intricate and must be attacked in the light of very meagre information. Hence, we must be careful not to substitute the comfortable certainties of some simple mathematical model in place of the great complexities of the actual universe. ... It is appropriate to approach the problems of cosmology with feelings of respect for their importance, of awe for their vastness, and of exultation for the temerity of the human mind in attempting to solve them. They must be treated, however, by the detailed, critical, and dispassionate methods of the scientist." R. Tolman ([1] p. 487-488).
\end{abstract}

Such non-carefulness, when "the great complexities of the actual universe" were replaced by "comfortable certainty in some simple mathematical model", as it turned out, was inherent in basic models of relativistic cosmology. If in the case of the static Einstein model [2] its excessive simplification was quickly clarified, then in the case of the non-static Friedmann model [3] that replaced it (see [4,5]) and the Standard Model of cosmology based on the latter (see [5]) it lasted almost hundred years. In the Friedmann model, the observed redshift in cosmology is attributed to the stretching of wavelengths during the expansion of space, while the contributions of the Doppler effect and gravitational redshift are ignored, naively believing that the relations of the model effectively contain them [4,5]. In the papers $[6,7]$ it was shown that this is "wish-fulfilling thinking" and the cost of this there was a number of contradictions in the model, which were eliminated by ignoring the contributions of the real effects and the introduction of artificial hypotheses instead (cosmological acceleration, dark energy etc.).

As it is known, in static space, close receding sources emit photons with the linear Doppler redshift. In [6] it was shown that in the expanding space this initial kinematical shift does not disappear anywhere and only the stretching redshift is added to it, but already on the way. As a result, photons from receding sources will arrive with at least doubled redshift in the linear part. For distant objects, the Doppler effect will become relativistic with a quadratic part, and a gravitational redshift of the same order will be added [7]. Thus, in the expanding space, the Friedman model with the linear element:

$$
d s^{2}=c^{2} d t^{2}-a^{2}(t) \cdot\left(d \chi^{2}+S_{k}^{2} d \Omega_{(2)}^{2}\right) .
$$

where $a=a(t), S_{k}(\chi)=(\sin \chi, 1, \sinh \chi)$ at $k=(1,0,-1)$, in the linear part leads to double redshift, while observations show a single redshift. For more distant objects, the growth with the distance of the redshifts should be even greater than with just stretching. Earlier, the solution to the double redshift problem was replaced by myths that the Doppler effect is just another way of describing the stretching of wavelengths, and the gravitational shift is supposedly contained in the formulas of the Friedmann model.

But agreement between theory and observations should be achieved not by ignoring one or another real physical phenomenon, but by excluding the explicit and hidden hypotheses of the Friedmann model, which gave rise to problems. One of these turned out to be a hidden hypothesis that the rate of proper time in every small region of the universe is constant and does not depend on time. This hypothesis was introduced to simplify the theory and was not confirmed by anything else.

In the paper [6], a model of slowing time cosmology (STC) was formulated without this artificial restriction, and it was shown that this more general model of relativistic cosmology solves the double redshift problem. In the present paper it will be also taken into account the gravitational shift for distant objects using a new consistent method described in [7]. In STC, both the spatial components of the metric and its temporal component change over time. The slowing down of proper times follows from the observational data, since it turned out that general relativity is consistent with observations only at a faster rate of proper times in the past and its slowing down during expansion. The linear element in terms of the time of our epoch $t$ then has the form: 


$$
d s^{2}=\frac{a_{0}^{2} f}{a^{2}} c^{2} d t^{2}-a \cdot\left(d \chi^{2}+S_{k}^{2} d \Omega_{(2)}^{2}\right),
$$

where $a_{0}=a\left(t_{0}\right)$ and $f(t)$ expresses gravitational time dilation, $f\left(t_{0}\right)=1$.

Thus, in STC there are three basic mechanisms of cosmological shift, which have linear parts - for nearby objects, along with the Doppler redshift and stretching, there is a violetshift of the frequencies of photons emitted in earlier epochs with a faster rate of proper times. This violetshift compensates for the stretching redshift along the path and as a result, the observed shift appears to be Doppler redshift. For distant objects, the contributions of the quadratic Doppler effect and gravitational shift will also be added, and the relativistic aberration [6] will reduce the apparent luminosities.

Some elements of STC have been previously studied by several authors for various reasons (the references see in [6]. Initially, there were many attempts to explain redshifts as the Doppler effect, sometimes including gravitational shift, but later the interpretation of the shift as stretching [4,5] became standard. Hypothetical models were also developed in which redshifts were explained only by a change in the light velocity. In contrast to these particular treatments, STC takes into account all these effects together - in it, the slowing down of the proper times rate leads to the slowing down of the light velocity as well, and both the Doppler effect and stretching contribute to the redshifts, but the contribution of the latter is compensated by the initial violetshift. The gravitational redshift contribution is of the same order of magnitude as the quadratic Doppler effect [7].

In the paper the main relations of STC are presented, including the "distance modulus redshift" relation. It is shown that STC is consistent with observations without hypotheses about dark energy and a large fraction of dark matter, but with different values of the cosmological parameters. In STC there are no cosmological problems of the previous models (horizon, homogeneity, flatness, cosmological constant, entropy and initial singularity) [6]. There are no problems with horizon and homogeneity because in early epochs the light velocity was faster and the radius of the horizon increased faster than at the current velocity of light.

In Section 1 two known mechanisms of cosmological redshift, kinematical and stretching, are considered and it is shown their combined action leads to the double redshift problem. In Section 2 two new shift mechanisms, the violetshift due to a faster rate of proper times in the past and the gravitational redshift, are discussed and it is shown the compensation of stretching redshift by the violetshift. In Section 3, the main relations of STC are presented and their observational consequences are considered taking into account all four kinds of the frequency shift and relativistic aberration. In Section 4 the early universe and CMB in STC are briefly discussed.

\section{Two redshift mechanisms and the double redshift problem}

\subsection{Kinematical redshift and wavelength stretching in cosmology}

Let an observer in a flat static space is located at $r=0$, and objects at distances $r$ have the receding velocity $v(r) \simeq H_{0} r$, where $H_{0}=$ const. Such receding sources emit photons with a wavelength $\lambda_{D}$ by the Doppler redshift $z_{D}$ relative to the wavelength $\lambda$ of photons from resting sources. At low velocities $v \ll c$ we have:

$$
\frac{\lambda_{D}}{\lambda}=1+z_{D} \simeq 1+\frac{v}{c} \simeq 1+\frac{H_{0} r}{c}, \quad r \simeq \frac{c}{H_{0}} z_{D},
$$

and for distant sources with high velocities, the relativistic Doppler effect gives:

$$
\frac{\lambda_{D}}{\lambda}=1+z_{D}=\sqrt{\frac{1+v / c}{1-v / c}}=\sqrt{\frac{1+H_{0} r / c}{1-H_{0} r / c}} .
$$


The distance to the source will then be expressed through $z_{D}$ as:

$$
r=\frac{v}{H_{0}}=\frac{c}{H_{0}} \cdot \frac{z_{D}+z_{D}^{2} / 2}{1+z_{D}+z_{D}^{2} / 2} .
$$

It is important here that the Doppler redshift $z_{D}$ from receding sources appears initially, at emission of photons, and then in static space these photons propagate with this constant wavelength $\lambda_{D}$.

Now let the space expands with the scale factor $a(t)$, where $r=a(t) \chi$, and at the moment of emission of the photon the source was at rest relative to the observer at $r=$ const., i.e. does not comove expansion. The velocity of such a resting source in our rest frame is zero: $\dot{r}=\dot{a} \chi+a \dot{\chi}=0$ and therefore its peculiar velocity $v_{\text {pec }}=a \dot{\chi}$ is equal in magnitude, but opposite in the direction to the receding velocity $v_{\text {rec }}=\dot{a} \chi=H r$ of the sources comoving the expansion: $v_{\text {pec }}=-v_{\text {rec }}$, where $H=\dot{a} / a, \dot{a}=d a / d t$. The periods of photons from such a source at the beginning $(\tau)$ and at the end of the path $\left(\tau_{E}\right)$, or the corresponding frequencies $\omega$ and $\omega_{E}$, according to (1), are given by the expressions:

$$
c \tau=a \cdot \Delta \chi, \quad c \tau_{E}=a_{0} \cdot \Delta \chi, \quad \frac{\tau_{E}}{\tau}=\frac{\omega}{\omega_{E}}=\frac{\lambda_{E}}{\lambda}=\frac{a_{0}}{a} .
$$

Thus, photons from the rested source emitted with the initial wavelength $\lambda=c / \tau$, arrive with the wavelength $\lambda_{E}$ increased proportionally $a(t)$, i.e. with stretching redshift $z_{E}$ :

$$
\frac{a_{0}}{a}=\frac{\lambda_{E}}{\lambda}=1+z_{E} .
$$

In the first approximation $z_{E}$ grows linearly with distance:

$$
a \approx a_{0} \cdot\left(1+H_{0} \Delta t\right)=a_{0} \cdot\left(1+\frac{H_{0} r}{c}\right), \quad z_{E} \simeq \frac{H_{0}}{c} r,
$$

which is similar to the linear Doppler effect (3).

Thus, the source rested relative to us (with a peculiar velocity $v_{p e c}=-H_{0} r$ relative to comoving the expansion bodies around it) emits photons without redshift, since there is no Doppler effect, but during the propagation of photons, they acquire the stretching redshift.

\subsection{Double redshift problem in expanding space}

Above, two mechanisms of redshift in general relativity were considered, when each of them manifests itself in a pure form, and the second is absent:

a. If the space is static, and the source is receding from us, then the photons are emitted with the Doppler redshift and will come with this redshift only.

b. If space expands, and the source is at rest relative to us (i.e., has a peculiar velocity $v_{\text {pec }}=a \dot{\chi}=-H_{0} r$ ), then photons are emitted without a Doppler shift, but their wavelength is stretched during propagation.

At small distances and velocities, the redshift is the same in both cases: $c z_{E} \simeq H_{0} r$, which was the reason for the confusion in its interpretation.

Consider now the third case, which is a combination of the two previous ones, and where both redshift mechanisms are present. This is the case when

c. the space expands and, in addition to the resting source (relative to us), there is also a receding source comoving the expansion. Its peculiar velocity $v_{\text {pec }}=a \dot{\chi}=0$, while the receding velocity: $\dot{r}=\dot{a} \chi+a \dot{\chi}=a \dot{\chi}=H_{0} r$. 
If the static source is slightly closer to us than the receding one, then for the observer near it, the photons from the receding source, receding with velocity $H_{0} r$, have a wavelength $\lambda_{D}$ with the Doppler redshift $z_{D}$ from (4). Thus, the Doppler redshift of photons from the receding source can be fixed in our rest frame at the beginning of the path.

Further, a photon from a resting source with a wavelength $\lambda$, as well as a photon from the receding source with a wavelength $\lambda_{D}$, having traveled almost the same path to us, will experience the same stretching of the wavelength, proportional to the growth of $a$. When the first photon arrives at us, its initial wavelength $\lambda$ becomes stretched and equal to $\lambda_{E}$ from (7), while the second photon with the initial wavelength $\lambda_{D}$ in our rest frame will experience the same stretching and come with the wavelength $\lambda_{D E}$, which follows from the analogue of (7):

$$
\frac{\lambda_{D E}}{\lambda_{D}}=\frac{a_{0}}{a}=1+z_{E} .
$$

Taking into account (4) and that it was $\lambda_{D}=\left(1+z_{D}\right) \lambda$ at the beginning, the total redshift for photons from the receding source is found as a combined redshift $z_{E D}$, expressed in terms of $z_{D}$ and $z_{E}$ as:

$$
\frac{\lambda_{D E}}{\lambda}=\frac{\lambda_{D E}}{\lambda_{D}} \frac{\lambda_{D}}{\lambda}=\left(1+z_{E}\right)\left(1+z_{D}\right)=1+z_{E D} .
$$

In the first approximation this gives:

$$
z_{E D} \simeq z_{E}+z_{D} \simeq 2 \frac{H_{0}}{c} r \simeq 2 \frac{v}{c}, \quad z \simeq 2 z_{E} \simeq 2 z_{D} .
$$

i.e. the contributions of the Doppler redshift $z_{D} \simeq r \cdot H_{0} / c$ and stretching redshift $z_{E} \simeq r \cdot H_{0} / c$ must be summed, which gives twice the total redshift than just one of these effects.

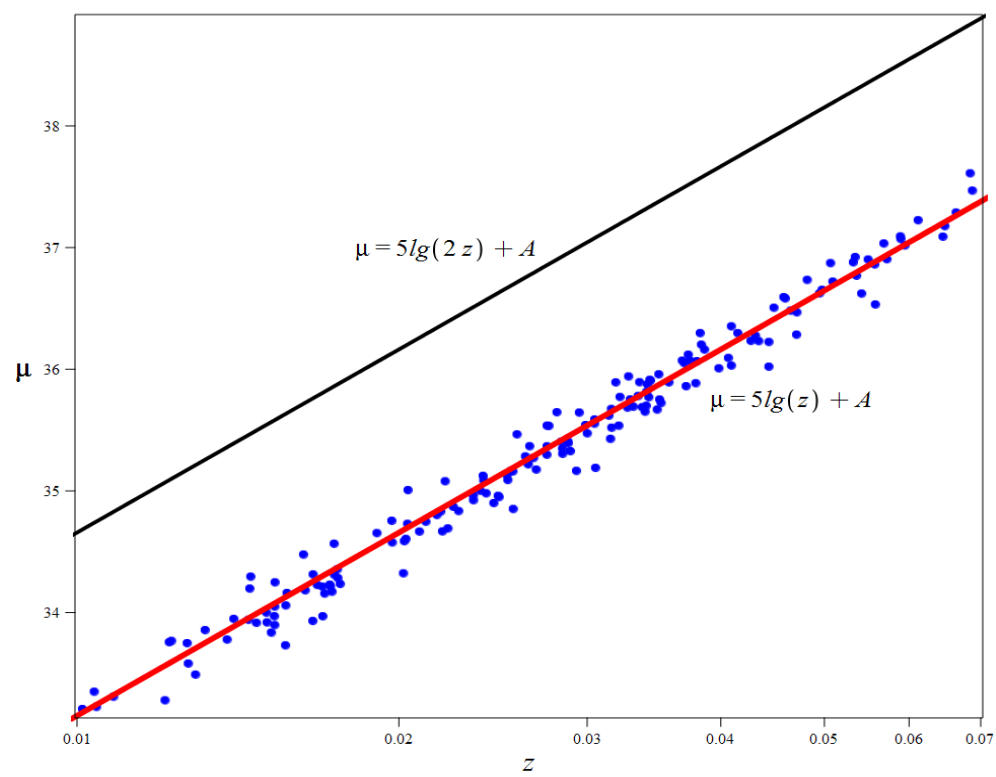

Fig. 1. Graph "distance modulus - redshift" for SN 1a in the linear part (on a logarithmic scale $z), A=5 \lg \left(c / H_{0}\right)+25, H_{0}=70 \kappa м / с е \kappa \cdot M n c$. Data from [10]. Black line - double redshift with $\delta \mu=5 \lg (2)=1.5$.

As shows Fig. 1, in fact there is a single effect according to the data and $z \simeq H_{0} r / c$. Therefore, in relativistic cosmology there was the double redshift problem, and previous models based on the Friedmann metric with two types of redshift in fact contradicted the observations. 


\section{Two new frequency shifting mechanisms and a solution to the double redshift problem}

\subsection{The initial violetshift compensating the stretching along the way}

In general relativity, therefore, the two basic mechanisms of cosmological redshift, the initial Doppler redshift and stretching redshift along the way, must be considered together and thus there is the double redshift problem. This means that in the Friedmann model there is some kind of unrealistic hidden assumption. As it is shown in [6], this is a strong constraint for the temporal component of the metric in the form of its constancy during the expansion, which means the hypothesis of the constancy of the proper time rate at each place.

In order to understand how strong such a restriction is, one must recall the fact that, according to the relativity theory, physical reality is not space and time separately, as it was in Newtonian theory, but is the $4 \mathrm{~d}$ space of events, or spacetime. For this reason, the cases when relativistic effects with space and time take place separately are very rare and exceptional. Therefore, firstly, when, during the expansion of space, the proper distance between two objects increased significantly, for example, 10 times, while the proper times in both places did not change and go at the same rate as before, it would be an exceptional case in relativistic systems. Secondly, such "independence" of proper time in each place from the behavior of space in the same place is characteristic for Newtonian theory and therefore it is not surprising that the relations of the Friedmann model are directly follow from the Newtonian cosmology.

In general relativity, from a physical point of view, the situation is more natural when a change in the spatial components of the metric, expressing a change in the curvature of space, leads to a corresponding change in the temporal component of the metric. If the latter changes in such a way that the rate of proper times in earlier epochs was higher than in the present, then the frequencies of the emitted photons would be higher than the present ones, i.e. they would be emitted by a violetshift. Then, to the two previous mechanisms, a third mechanism would also be added - the violetshift at the emission of photons in earlier epochs. It would compensate for the stretching redshift along the path and, as a result, only a single Doppler redshift would be observed in the linear part, which would naturally agree the theory with the observations.

In earlier models, the wavelength $\lambda$ of a photon emitted in earlier epochs was assumed to be the same as the wavelength $\lambda_{0}$ of a photon emitted here now, at the time of reception. However, in reality, general relativity, together with the cosmological principle, requires only that the wavelength of a photon when it is emitted at an earlier epoch $\tau(\chi)=\tau_{1}$ coincides with the wavelength of a similar photon near the observer only at the same early epoch, i.e. in their proper time $\tau(0)=\tau_{1}$ (Fig. 2). For example, the wavelength of a photon emitted far away from us in the epoch of 10 billion years after the Big Bang local time there should coincide with the wavelength of the same photon here, also in the epoch of 10 billion years after the Big Bang local time.

After the comparison of the

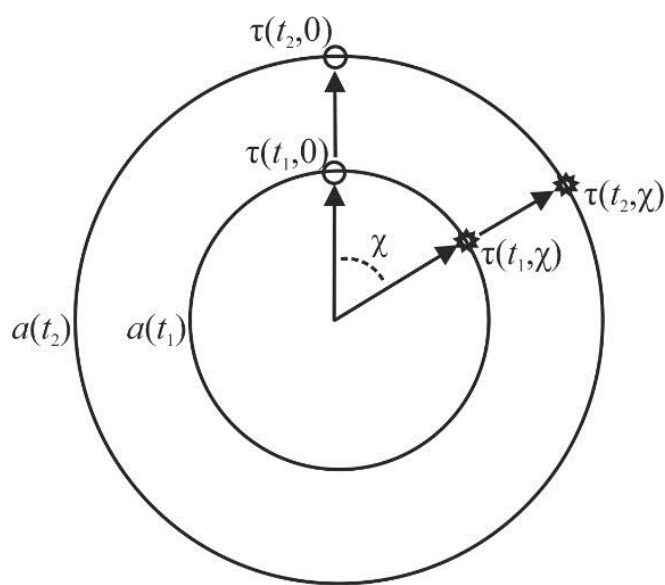

Fig. 2. An example of the proper time rate in a closed universe. The cosmological principle requires that the proper time rate in different places be the same in the same epoch and allows for different time rates in different epochs. wavelengths of the photons at the place of emission and at the place of reception is carried out correctly, namely, in terms of the wavelengths at these places in the same cosmological epoch, 
another problem arises - how the photon wavelength is related to us in that epoch with the wavelength of the same photon in our epoch.

Since the proper times in STC are variable, it is necessary to choose the time of one of the epochs as a standard, and the times of other epochs to express in its terms as time coordinates. Our time is a natural choice for us, since signals from all previous epochs are compared with signals in our epoch. Then it is convenient to express the proper times $\tau$ in terms of our local proper time $t$ :

$$
d \tau^{2}=g_{00}[a(t)] d t^{2} .
$$

In general relativity, the choice of the time component of the metric $g_{00}$ depends on physical conditions and this metric can be either less or more than unity: $g_{00}[a(t)]<1$ or $g_{00}[a(t)]>1$. Observational facts show that the double redshift problem is solved only if, during the cosmological expansion, the rate of local proper times was greater in previous epochs and it slowed down.

Is there a similar case in relativistic systems when, as a free particle evolves along the world line, the surrounding space would expand, and the rate of the particle's proper time would become slower than in the previous sections of the same world line? Yes, there is such a system and it is the Schwarzschild field, i.e. a static gravitational field around a spherical source. As a radially falling free particle crosses spheres with circumferential radii $r_{1}, r_{2}, \ldots$ differing by $\Delta r$ , the intervals of the physical distance $\Delta \mathrm{r}$ along the radius between two neighbor spheres increase as $\Delta \mathrm{r}=\Delta r /\left(1-r_{g} / r\right)^{1 / 2}$, i.e. the space around the falling particle "expands" in the radial direction. The intervals of the proper time of the particle $\Delta \tau$ become smaller in comparison with the same intervals of the world time $\Delta t$, i.e. proper time slows down as $\Delta \tau=\Delta t\left(1-r_{g} / r\right)^{1 / 2}$ . In this case, the coefficient at $\Delta t$ is inverse to the coefficient at $\Delta r$.

During cosmological evolution, the space around a particle also expands, but not in one dimension, but in all three dimensions and with the coefficient $a(t) / a\left(t_{0}\right)$ at $\Delta r$, where $\Delta r$ expresses the physical distances in our epoch. If we follow to the analogy with the fall in the Schwarzschild field, then the proper times should also slow down during expansion and the coefficient at $\Delta t$ should be the inverse to the coefficient at $\Delta r$, i.e. equal to $a\left(t_{0}\right) / a(t)$.

Thus, the dependence of the rate of proper times on the scale factor, as the analogy with the Schwarzschild field shows, is possibly the same as for stretching, i.e. the proper time interval multiplied to the scale factor remains unchanged:

$$
a d \tau=a_{0} d t, \quad d \tau=\frac{a_{0}}{a} d t .
$$

For the metric in (12), this means that $g_{00}(t)=a_{0}^{2} / a^{2}$, i.e. for $a<a_{0}$ we have $g_{00}(t)>1$.

Since the rate of proper time was faster, the frequencies of the photons in each place were higher and the wavelengths were less than those of the same photons now. This leads to a violetshift in earlier epochs with a factor inverse to the stretching factor in (9). Therefore, if the wavelength of a photon during emission should have been equal to $\lambda_{D}$ only at taking into account the Doppler effect, then in fact the wavelength was shorter due to the initial violetshift. Denoting this wavelength as $\lambda_{C D}$, we can find it from the relationship:

$$
\frac{\lambda_{C D}}{\lambda_{D}}=\frac{a}{a_{0}}=\frac{1}{1+z_{E}} .
$$

Then the initial violetshift due to the faster time rate in (14) and the further redshift due to stretching from (9) compensate each other and, as a result, the cosmological shift $1+z_{C D E}$, taking into account three effects, is reduced to the Doppler effect from (4). The corresponding wavelength $\lambda_{C D E}$ is then calculated as the result of a chain of ratios: 


$$
1+z_{C D E}=\frac{\lambda_{C D E}}{\lambda_{0}}=\frac{\lambda_{C D E}}{\lambda_{D E}} \cdot \frac{\lambda_{D E}}{\lambda_{0}}=\frac{\lambda_{C D E}}{\lambda_{D E}} \cdot \frac{\lambda_{D E}}{\lambda_{D}} \frac{\lambda_{D}}{\lambda_{0}}=\frac{a}{a_{0}} \cdot \frac{a_{0}}{a} \frac{\lambda_{D}}{\lambda_{0}}=\frac{\lambda_{D}}{\lambda_{0}}=1+z_{D} .
$$

Thus, we obtain the exact relations:

$$
1+z_{C D E}=\frac{\lambda_{C D E}}{\lambda_{0}}=\frac{\lambda_{D}}{\lambda_{0}}=1+z_{D}, \quad \lambda_{C D E}=\lambda_{D}, \quad z_{C D E}=z_{D} .
$$

In the first approximation, this gives the linear Doppler effect, which from the very beginning was considered to be an explanation of the observed linear dependence of $z$ on $r$ and $v$ :

$$
z_{C D E}=z_{D} \simeq r \cdot H_{0} / c \simeq v / c .
$$

Thus, the double redshift problem in a linear part, which consists in the combined action of the Doppler effect and stretching during the expansion of space, is solved in STC in a natural way. The previous formulation contradicted observations already for close objects, where the conclusions are model independent, since only the cosmological parameter $H_{0}$ plays a role and there are no kinematical relativistic effects.

\subsection{Gravitational time dilation and its contribution to redshift}

Another fundamental physical phenomenon that follows from general relativity and must be taken into account in cosmology is gravitational time dilation. It leads to gravitational redshift in cosmology (GRC), which grows in proportion to the square of the distance and is significant for distant objects. However, as it was shown in [7], two previous attempts to take it into account were erroneous, and a consistent theory of two types of GRC in cosmology was formulated.

The first type, local GRC, is the result of the influence of gravity of matter in the sphere between the observer and the source, centered in the middle of the distance to the source (Fig. 2). At the moment of emission, the source and the observer are on the periphery of this sphere at the same distance from its center, and in the static world the exchange of photons between them does not lead to GRC. But in the expanding world, the photons emitted at a smaller radius of this sphere and stronger gravity are received by the observer at a larger radius with the same mass of the sphere, when the gravity on its surface has become weaker, which leads to the local GRC, depending on the difference in radii.

The influence of gravity of the matter of the outer region is similar and leads to the global $G R C$ due to the difference in average densities, and hence the dilation of proper times, in two epochs. The photons from the observer to the source have the same two types of GRC in full agreement with the cosmological principle.

Let the sphere between the observer and the source be an empty cavity. Then the gravitational potential created in it by all matter of the universe will be constant in the entire cavity at every moment, but changes over time due to a decrease in the average density of the matter in the universe. In a static frame of reference with spherical coordinates centered at the center of the cavity, we introduce a hypersurface of simultaneity $t=$ const. with world time $t$. The linear element then includes a function $f(t)$ depending on the average density $\rho(t)$ of the matter outside the cavity:

$$
d s^{2}=f(t) d t-d r^{2}-r^{2} d \Omega^{2} .
$$

In a static world with constant density $\rho=$ const., it would be $f=$ const., which allows to redefine world time as $f \cdot d t^{2}=d t^{\prime 2}$. In this case, $t^{\prime}$ would also be world time, since for simultaneous events its values would be the same. In a static world, therefore, there is no global GRC. In the expanding world, where during the propagation of photons from the source inside 
the cavity $\rho(t)$ will decrease, and the radius of the cavity will increase (Fig. 2), the value of $f(t)$ will increase and the gravitational dilation of proper times will weaken: $f\left(t_{2}\right)>f\left(t_{1}\right)$ at $t_{2}>t_{1}$.

At the first stage, we will determine the value of GRC for this reason in its pure form, excluding the Doppler shift. To do this, consider the situation when the source at emission and the observer at receiving of photons were at rest relative to the center of the cavity. Since $\tau_{o}=\Delta t \sqrt{f\left(t_{o}\right)}$ and $\tau_{e}=\Delta t \sqrt{f\left(t_{e}\right)}$, then for the global GRC we get:

$$
1+z_{g G}=\frac{\lambda_{o}}{\lambda_{e}}=\frac{\tau_{o}}{\tau_{e}}=\frac{\sqrt{f\left(t_{o}\right)}}{\sqrt{f\left(t_{e}\right)}}
$$

At the second stage, we will take into account the Doppler shift and consider the emission and registration of photons by the source and observer comoving the surface. At the rate of expansion of the cavity's boundary relative to its center $v_{r}=H r$, the relative velocity of the source and the observer on the hypersurfaces of simultaneity $t_{e}=$ const. and $t_{o}=$ const. is determined by the relativistic formula, which, in the case when $v_{r}$ weakly depends on time, gives:

$$
v=\frac{2 H r}{1+H^{2} r^{2}}, \quad 1+z_{D}=\sqrt{\frac{1+v}{1-v}}=\frac{1+H r}{1-H r}=\frac{1+H_{o} r_{o} / 2}{1-H_{o} r_{o} / 2},
$$

Thus, the total shift in this approximation takes the form:

$$
1+z_{g G, D}=\left(1+z_{g G}\right)\left(1+z_{D}\right)=\frac{\sqrt{f\left(t_{o}\right)}}{\sqrt{f\left(t_{e}\right)}} \cdot \frac{1+H_{o} r_{o} / 2}{1-H_{o} r_{o} / 2} .
$$

Thus, the global GRC arises due to the fact that photons are emitted in an epoch with a higher average density and are received in an epoch with a lower average density. The gravity of denser matter slowed down its proper times stronger and photons were created with a lower frequency than at present, which leads to GRC of "old" photons relatively "young" ones.

To determine the local GRC in its pure form, consider the case opposite to considered in the previous Section - let the cavity between the observer and the source be filled with a homogeneous dust matter, and there is no matter outside the cavity, i.e. let us take into account the gravity of the sphere matter, but let's exclude the influence of matter outside the sphere.

In the static world, photons from a source at the periphery of the gravitating sphere come to the observer at the diametrically opposite periphery at the same radius of the sphere. Since there is no potential difference between these points, there will be no gravitational shift.

In the expanding world, the gravitational shift at the periphery of the sphere will also be studied at first without the contribution of the Doppler shift, when the source is at emission and the observer at receiving is at rest relative to the center of the sphere between them. Now, during the propagation of the photon, the sphere will expand and the photons emitted at a smaller radius will come to the observer on the sphere at a larger radius, when its gravity has weakened. The ratio of the time components of the Schwarzschild metric (or the potential difference in the case of a weak field), will lead to a local GRC for the photon frequencies.

Thus, photons emitted at the radius of the sphere $r\left(t_{e}\right)$ come to the observer at a larger radius $r\left(t_{o}\right)>r\left(t_{e}\right)$ at the same mass of the sphere $M_{e} / 8$, which leads to the local GRC. In a static world with the source and observer at rest, they would have:

$$
1+z_{g L}=\frac{\lambda_{o}}{\lambda_{e}}=\sqrt{\frac{1-2 G M(r) / r\left(t_{e}\right)}{1-2 G M(r) / r\left(t_{o}\right)}}=\sqrt{\frac{1-H_{e}^{2} r_{e}^{2} / 4}{1-H_{0}^{2} r_{o}^{2} / 4}} \simeq 1+\frac{1}{8}\left(H_{0}^{2} r_{o}^{2}-H_{e}^{2} r_{e}^{2}\right) .
$$


Accordingly, the photons from the source, which in the observer's rest frame was receded with a velocity $v$, at the moment of emission contain the Doppler redshift $1+z_{D}$ as in (20) and therefore the combined shift $1+z_{g L, D}$ will be equal to:

$$
1+z_{g L, D}=\left(1+z_{g L}\right)\left(1+z_{D}\right)=\frac{\lambda_{o}}{\lambda_{e}}=\frac{1+H_{o} r_{o} / 2}{1-H_{o} r_{o} / 2} \cdot \frac{\sqrt{1-H_{e}^{2} r_{e}^{2} / 4}}{\sqrt{1-H_{o}^{2} r_{o}^{2} / 4}} .
$$

Thus, the maximal value of the local GRC, which occurs at $r_{o} \gg r_{e}$, is approximately half of the quadratic (or transverse) Doppler redshift.

Local and global GRC from (19) and (22) together form a total GRC $1+z_{g}$, which is their product:

$$
1+z_{g}=\left(1+z_{g G}\right)\left(1+z_{g L}\right) .
$$

Taking into account the initial Doppler effect (20) gives the combined shift $1+z_{g, D}$ :

$$
1+z_{g D}=\left(1+z_{g}\right)\left(1+z_{D}\right) .
$$

Thus, GRC is similar to the quadratic Doppler effect and is not contained in the relations of the Friedmann model, as it was commonly believed [4]. Therefore, GRC should be taken into account separately both in the models and in the interpretation of data for distant objects. Thus, the theory of GRC makes serious modifications both to cosmological models and their predictions, and to the interpretation of observational data.

Of the two types of GRC, the theory of local shift for photons from observable objects does not require new hypotheses and its consequences can be used to correct the data in the form of corrections for this part of the effect. The situation with the global GRC is more complicated, since its calculations require knowledge of the structure of the universe on a global scale. Since the global GRC is sensitive to the choice of the cosmological model, this feature makes it a valuable tool for testing cosmological models.

In the early universe, the contributions of both types of GRC already differ little and significantly modify the previous picture concerning the distances to objects, their age and evolution, as well as about the properties of CMB.

\subsection{Observed redshifts as a result of the Doppler effect and GRC}

In Section 1 it was shown that in the expanding space the photons from the comoving the expansion sources come with two types of redshift - the Doppler and stretching, and in Section 2.2. it was shown that for sufficiently distant objects, another type of redshift will be added GRC. These three types of redshift are independent of each other and must be taken into account in any of the cosmological models with expanding space.

The models based on the Friedmann model, containing only redshifts, turned out to be oversimplified and inconsistent with reality, since even for close objects they lead to a double redshift in comparison with what observations show.

In STC, as shown in Section 2.1, there is an initial violetshift due to the faster time rate in (14) and then it compensates for the stretching redshift from (9). As a result, the observed redshift is reduced to the contributions of the Doppler effect from (4) and GRC from (24), i.e. has the form (25).

The observed wavelength $\lambda=\lambda_{C D E g}$, including the contribution of all four effects, is then calculated as the result of a chain of relationships: 


$$
1+z=\frac{\lambda_{C D E g}}{\lambda_{0}}=\frac{\lambda_{C D E g}}{\lambda_{D E g}} \cdot \frac{\lambda_{D E g}}{\lambda_{0}}=\frac{\lambda_{C D E g}}{\lambda_{D E g}} \cdot \frac{\lambda_{D E g}}{\lambda_{D g}} \frac{\lambda_{D g}}{\lambda_{0}}=\frac{\lambda_{C D E g}}{\lambda_{D E g}} \cdot \frac{\lambda_{D E g}}{\lambda_{D g}} \cdot \frac{\lambda_{D g}}{\lambda_{g}} \cdot \frac{\lambda_{g}}{\lambda_{0}},
$$

which gives:

$$
1+z=\frac{a}{a_{0}} \cdot \frac{a_{0}}{a} \cdot\left(1+z_{D}\right)\left(1+z_{g}\right)=\left(1+z_{D}\right)\left(1+z_{g}\right)=1+z_{g D} .
$$

For close objects, thus, a linear Doppler shift will be observed, and for sufficiently distant objects, the quadratic Doppler effect and GCS of the same order become added.

\section{Slowing time cosmology (STC) taking into account the effects of gravity}

\subsection{Basic relationships of the model}

The double redshift problem in relativistic cosmology is solved only in STC, and STC then modifies the description of the structure and evolution of the universe. The formulation of STC in [6] was given without taking into account GRC, and an estimated comparison with observations showed that, in the first approximation, the model agrees with the data. In this section, this formulation will be generalized by taking into account the gravitational time dilation and some of consequences will be clarified, including comparisons with observations.

In Friedmann's models, in the comoving frame of reference, the local linear element was written in terms of proper time $\tau$. In STC, the linear element has the form (2), i.e. in accordance with (13) and (18), we write in terms of time of present epoch $t$ as $d \tau=d t \cdot \sqrt{f} a_{0} / a$. Then the time component and the determinant of the metric take the form:

$$
g_{00}(t)=\frac{a_{0}^{2} f(t)}{a^{2}(t)}, \quad \sqrt{-g}=a_{0} a^{2} \sqrt{f}|\sin \theta| S_{k, \chi}^{2} .
$$

The energy-momentum tensor of homogeneous dust with local energy density $\rho(t)$ :

$$
T^{i k}=\rho \frac{d x^{i}}{d s} \frac{d x^{k}}{d s}, T^{00}=\rho \frac{a^{2}}{a_{0}^{2} f}, \quad T_{0}^{0}=T^{00} g_{00}=\rho, \quad T_{00}=\rho g_{00}=\rho f \frac{a_{0}^{2}}{a^{2}}
$$

and the energy-momentum conservation condition give the former relations $(\dot{a}=d a / d t)[4]$ :

$$
\frac{1}{a^{2}} \frac{\partial}{\partial t}\left(\rho a^{2}\right)+\rho \frac{\dot{a}}{a}=0, \quad \frac{\partial}{\partial t}\left(\rho a^{3}\right)=0, \quad \rho a^{3}=\rho_{0} a_{0}^{3} .
$$

The Einstein equations $G_{i k}=\left(8 \pi G / c^{4}\right) T_{i k}$ with the Einstein tensor $G_{i k}$ and (30) give the evolution equation:

$$
\dot{a}^{2}=c^{2} \frac{a_{0}^{2} f}{a^{2}}\left(\frac{a_{m}}{a}-k\right), \quad \dot{a}= \pm c \frac{a_{0} \sqrt{f}}{a} \sqrt{\frac{a_{m}}{a}-k}, \quad a_{m} \equiv \frac{8 \pi G \rho_{0} a_{0}^{3}}{3 c^{4}} .
$$

Comparison with Friedmann's equation:

$$
\left(\frac{d a}{d \tau}\right)^{2}=c^{2}\left(\frac{a_{m}}{a}-k\right), \quad \frac{d a}{d \tau}= \pm c \sqrt{\frac{a_{m}}{a}-k},
$$

shows that the expansion rate in (31) contains a new factor $\sqrt{f} a_{0} / a$. On the one hand, presence of $a_{0} / a$ means a faster expansion rate in earlier epochs in terms of $t$. On the other hand, the factor of gravitational time dilation $\sqrt{f}$ begins to increase for sufficiently distant objects, 
slowing down the processes and becomes dominant for the early universe. The light velocity $\tilde{c}$ was also variable:

$$
\tilde{c}^{2} d t^{2}-a^{2} d \chi^{2}=0, \quad \tilde{c}=\frac{a_{0} \sqrt{f}}{a} c .
$$

For our epoch with $a_{0}$ and constant $H_{0}$ Eq. (31) gives the standard expressions:

$$
\dot{a}_{0}= \pm c \frac{\sqrt{1-k a_{0} / a_{m}}}{\sqrt{a_{0} / a_{m}}}= \pm c \frac{\sqrt{1-k b}}{\sqrt{b}}, \quad H_{0}=\frac{\dot{a}_{0}}{a_{0}}= \pm \frac{c}{a_{0}} \frac{\sqrt{1-k b}}{\sqrt{b}}, \quad b \equiv \frac{a_{0}}{a_{m}} .
$$

For proper time $\tau$ from (32) we obtain:

$$
c \tau= \begin{cases}c \tau=\int_{0}^{a(t)} \frac{a^{1 / 2} d a}{\sqrt{a_{m}-k a}}, & k=0 \\ \frac{\sqrt{a\left(a_{m}-a\right)}+a_{m} \arcsin \sqrt{\frac{a}{a_{m}}},}{3 a_{m}^{1 / 2},} & k=1 \\ \sqrt{a\left(a_{m}+a\right)}-a_{m} \operatorname{arcsh} \sqrt{\frac{a}{a_{m}}}, & k=-1\end{cases}
$$

For time $t$, corresponding to $a(t)$, the evolution equation (31) gives

$$
\begin{aligned}
& c t=\frac{1}{a_{0} \sqrt{f}} \int_{0}^{a(t)} \frac{a^{3 / 2} d a}{\sqrt{a_{m}-k a}} \\
& c t= \begin{cases}\frac{3 a_{m}}{4 a_{0} \sqrt{f}}\left[a_{m} \arcsin \sqrt{\frac{a}{a_{m}}}-\left(1+\frac{2 a}{3 a_{m}}\right) \sqrt{a\left(a_{m}-a\right)}\right], & k=1 \\
\frac{2}{3} \frac{a^{3 / 2}}{a_{m}^{1 / 2}} \frac{3 a}{5 a_{0} \sqrt{f}}=\frac{3 a}{5 a_{0} \sqrt{f}} \tau, & k=0 \\
\frac{3 a_{m}}{4 a_{0} \sqrt{f}}\left[a_{m} \operatorname{arcsh} \sqrt{\frac{a}{a_{m}}}-\left(1-\frac{2 a}{3 a_{m}}\right) \sqrt{a\left(a_{m}+a\right)}\right], & k=-1\end{cases}
\end{aligned}
$$

\section{2. «Distance modulus - redshift» relation}

The world line of photons from distant sources is described by the ratios:

$$
\frac{a_{0}^{2} f}{a^{2}} c^{2} d t^{2}-a^{2} d \chi^{2}=0, \quad c d t= \pm \frac{a^{2}}{a_{0} \sqrt{f}} d \chi .
$$

For the angle $\chi$ passed by the photon, taking into account (31), this gives:

$$
\chi=c \int_{t}^{t_{0}} \frac{a_{0} \sqrt{f}}{a^{2}} d t=c \int_{t}^{t_{0}} \frac{a_{0} \sqrt{f}}{a^{2}} \frac{d a}{\dot{a}}=\int_{a}^{a_{0}} \frac{d a}{\sqrt{a\left(a_{m}-k a\right)}} .
$$

Integration eventually gives the expression of the Friedmann model:

$$
a_{0} S_{k}(\chi)=\frac{c}{H_{0}} \frac{a}{a_{0}} \cdot 2(1-k b)\left[\frac{a_{0}}{a}-1+(2 k b-1)\left(\sqrt{1+\frac{a_{0} / a-1}{1-k b}}-1\right)\right] .
$$

The ratio $a_{0} / a$ gives the magnitude of the stretching of wavelengths: 


$$
\frac{a_{0}}{a}=1+z_{E}
$$

and substitution of this into (41) gives:

$$
a_{0} S_{k}(\chi)=\frac{c}{H_{0}\left(1+z_{E}\right)} \frac{1}{q_{0}}\left[z_{E}+\left(q_{0}^{-1}-1\right)\left(\sqrt{1+2 q_{0} z_{E}}-1\right)\right] .
$$

where $2 q_{0}=1 /(1-k b)$. This is Mattig's formula [9] for stretching redshift. In the Friedmann model, which reduces the cosmological redshift only to stretching, this formula was directly compared with observations. It did not take into account both the Doppler effect and aberration, and the gravitational shift, since when these effects were taken into account, the problem of double redshift arose already in the linear part.

In STC, the total shift $z$ is associated with the Doppler effect (4) and GRC, in the calculation of the contribution of which there are also terms that include $a_{0} / a$, and therefore $1+z_{E}$ also.

First, we will consider relativistic effects in static space, when on the hypersurface of simultaneity $t=$ const. the sources recede with velocities proportional to the distance $v=H_{0} r$. Using this example, we will clarify which of the definitions of the distance to use in further calculations.

The fact is that even in this simple case, there are different types of distances, each of which has its own specific physical meaning. First, this is the luminosity distance $r$, equal to the radius of curvature of the sphere, to which the radiation spreads at the receiving time $t_{0}$. Secondly, the physical distance $r$, consisting of a set of standard scales comoving the sources, which are stick together simultaneously at the moment $t_{0}$ :

$$
\mathrm{r}=\int_{0}^{r} \frac{d r}{\sqrt{1-H_{0}^{2} r^{2} / c^{2}}}=\frac{c}{H_{0}} \arcsin \left(\frac{H_{0} r}{c}\right), \quad H_{0} r=c \sin \left(\frac{H_{0} \mathrm{r}}{c}\right) .
$$

Although in static space it makes no sense to operate with this "distance", but it can be determined unambiguously, and its analogue in the non-static case acquires practical meaning.

In an expanding curved space, where the physical distance is equal to $\mathrm{r}(t, \chi)=a(t) \chi$, the situation is more complicated. The local receding velocity of objects $\mathrm{v}(t, \chi)$ depends on both distance and time, and we deal with $\mathrm{H}(\tau)=a^{-1} d a / d \tau$ instead of $H_{0}$. Here there is only a local law for the difference in the receding velocities $\delta \mathrm{v}$ of two close objects, the physical distance between which at the moment $t$ is equal to $\delta \mathrm{r}$. From the definition of this distance

$$
\delta \mathrm{r}(t, \chi)=\mathrm{r}(t, \chi+\delta \chi)-\mathrm{r}(t, \chi)=a(t) \delta \chi
$$

we find the receding velocity of matter in this spatial area in the form:

$$
\delta \mathrm{v}(t, \chi)=\frac{\partial}{\partial \tau} \delta \mathrm{r}(t, \chi)=\mathrm{H} \cdot a \delta \chi
$$

which gives the required the local law of receding:

$$
\delta \mathrm{v}(t, \chi)=\mathrm{H} \cdot \delta \mathrm{r}(t, \chi) .
$$

In relativistic cosmology, the rates of receding of close objects on the hypersurface $t=$ const. are related by the relativistic relation: 


$$
\mathrm{v}(\chi+\delta \chi)=\frac{\mathrm{v}(\chi)+\mathrm{H} \delta \mathrm{r}}{1+\mathrm{v}(\chi) \cdot \mathrm{H} \delta \mathrm{r} / c^{2}},
$$

which, due to the smallness of $\delta \mathrm{v} / c$, can be written as

$$
\mathrm{v}(\chi+\delta \chi)-\mathrm{v}(\chi) \simeq\left[1-\mathrm{v}^{2}(\chi) / c^{2}\right] \mathrm{H} a \cdot \delta \chi .
$$

From this, taking into account (44), we obtain an equation for determining the local velocities:

$$
\frac{d \mathrm{v}}{1-\mathrm{v}^{2} / c^{2}}=\mathrm{H} d \mathrm{r}=\mathrm{H} \frac{d r}{\sqrt{1-\mathrm{v}^{2} / c^{2}}},
$$

which gives the dependence of the local velocity of receding from the distance (in the approximation when the change of $\mathrm{H}$ along the hypersurface $t=$ const. is neglected):

$$
r(t) \simeq \frac{c}{\mathrm{H}} \int_{0}^{\mathrm{v}(t, x)} \frac{d(\mathrm{v} / c)}{\sqrt{1-\mathrm{v}^{2} / c^{2}}}=\frac{c}{\mathrm{H}} \arcsin \left(\frac{\mathrm{v}}{c}\right), \quad \mathrm{v}=c \sin \left(\frac{\mathrm{H} r}{c}\right) .
$$

The photons from the comoving the expansion sources arrive at us with the same Doppler redshift $\left(1+z_{D}\right)$ that was already at the beginning of their path. Therefore, (4) contains exactly $\mathrm{v}$, which allows one to find a formula for the dependence of $r$ on $z_{D}$ :

$$
r=\frac{c}{H_{0}} \frac{H_{0}}{\mathrm{H}} \arcsin \left(\frac{z_{D}+z_{D}^{2} / 2}{1+z_{D}+z_{D}^{2} / 2}\right) .
$$

Since $\mathrm{H}$ is proportional to $H_{0}$ and depends on the factor $a_{0} / a=\left(1+z_{E}\right)$, then:

$$
\frac{H_{0}}{\mathrm{H}}=\frac{\dot{a}_{0}}{\dot{a}} \frac{a}{a_{0}}=\frac{a^{2}}{a_{0}^{2}} \frac{\sqrt{f_{0}}}{\sqrt{f}} \frac{\sqrt{1-k b}}{\sqrt{1-k b+a_{0} / a-1}}=\frac{1+z_{g}}{\left(1+z_{E}\right)^{2} \sqrt{1+2 q_{0} z_{E}}},
$$

At small distances $z_{D}, z_{E} \ll 1$ and (51) turns into a linear law:

$$
\mathrm{v} \simeq H_{0} r \simeq c z_{D} .
$$

The apparent and absolute luminosities $l, L$ of sources are related to the photometric distance $d_{p}$ as $l=L / 4 \pi d_{p}^{2}$. Through the apparent and absolute magnitudes $m$ and $M$ they are expressed as $l=10^{-m / 2.5} \cdot 2.52 \cdot 10^{-5} \mathrm{erg} / \mathrm{cm}^{2} \mathrm{sec}$ and $L=10^{-M / 2.5} \cdot 3.02 \cdot 10^{35} \mathrm{erg} / \mathrm{sec}$. Expansion leads to a decrease in the energy and frequency of arrival of photons by a total value $\left(1+z_{D}\right)^{2}\left(1+z_{g}\right)^{2}$ , and relativistic aberration reduces the apparent luminosity by a factor $\left(1+z_{D}\right)^{2}$. As a result, we obtain the expression for the apparent luminosity:

$$
l_{F}=\frac{L}{4 \pi d_{p}^{2}}=\frac{L}{4 \pi r^{2}} \cdot \frac{1}{\left(1+z_{D}\right)^{4}\left(1+z_{g}\right)^{2}} .
$$

Thus, the photometric distance $d_{p}$ is equal to:

$$
d_{p, 0}=\left(1+z_{D}\right)^{2}\left(1+z_{g}\right) r=10^{-5+(m-M) / 5} M n c,
$$

whence, taking into account the definition of the distance modulus $\mu \equiv m-M=5 \lg \left(d_{p}\right)+25$ and (52), the "distance modulus - redshift" relation follows $\left(A \equiv 5 \cdot \lg \left(c / H_{0}\right)+25\right)$ :

$$
\mu=5 \lg \left(\frac{\left(1+z_{D}\right)^{2}\left(1+z_{g}\right)^{2}}{\left(1+z_{E}\right)^{2} \sqrt{1+2 q_{0} z_{E}}} \arcsin \left(\frac{z_{D}+z_{D}^{2} / 2}{1+z_{D}+z_{D}^{2} / 2}\right)\right)+A .
$$


Slowing time cosmology with initial violetshift and three types of redshift

15

The quantity $\left(1+z_{E}\right)$ is proportional to the distance, and hence to the receding velocity of an object, while $1+z_{D}$ depends on the velocity not only linearly, but together with $1+z_{g}$ has a quadratic dependence on $r$ at large distances and therefore $z_{D}>z_{E}$. Expression $z_{E}$ through $z_{D}$ will significantly complicate the form (57), while in order to estimate the extent to which STC can be in agreement with the observational data, we need to simplify this formula as much as possible. To do this, at $z_{D}<2$, we approximate the multiplier in front of arcsin by using a small parameter $\alpha$ :

$$
\frac{\left(1+z_{D}\right)^{2}\left(1+z_{g}\right)}{\left(1+z_{E}\right)^{2} \sqrt{1+2 q_{0} z_{E}}} \simeq\left(1+z_{D}\right)^{1+\alpha z_{D}} .
$$

Substituting this into (57) and denoting $z_{D}$ as $z$, we obtain the desired simplified version of the "distance modulus - redshift" relation in STC [6]:

$$
\mu=5 \lg \left((1+z)^{1+\alpha z} \arcsin \left(\frac{z+z^{2} / 2}{1+z+z^{2} / 2}\right)\right)+A
$$

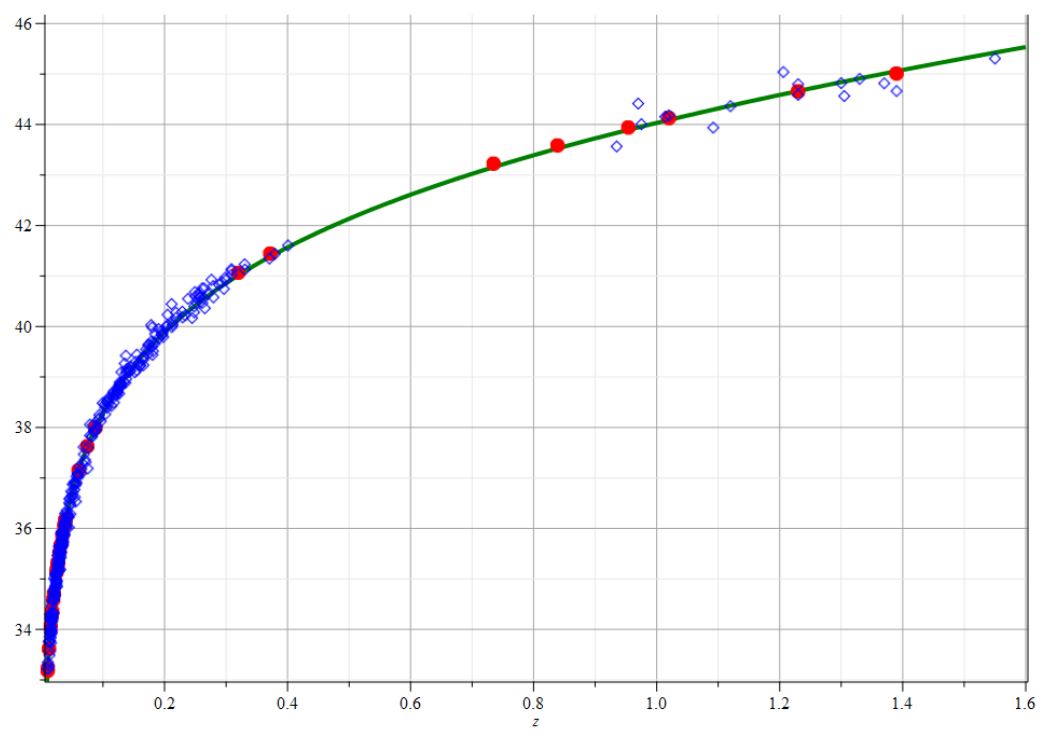




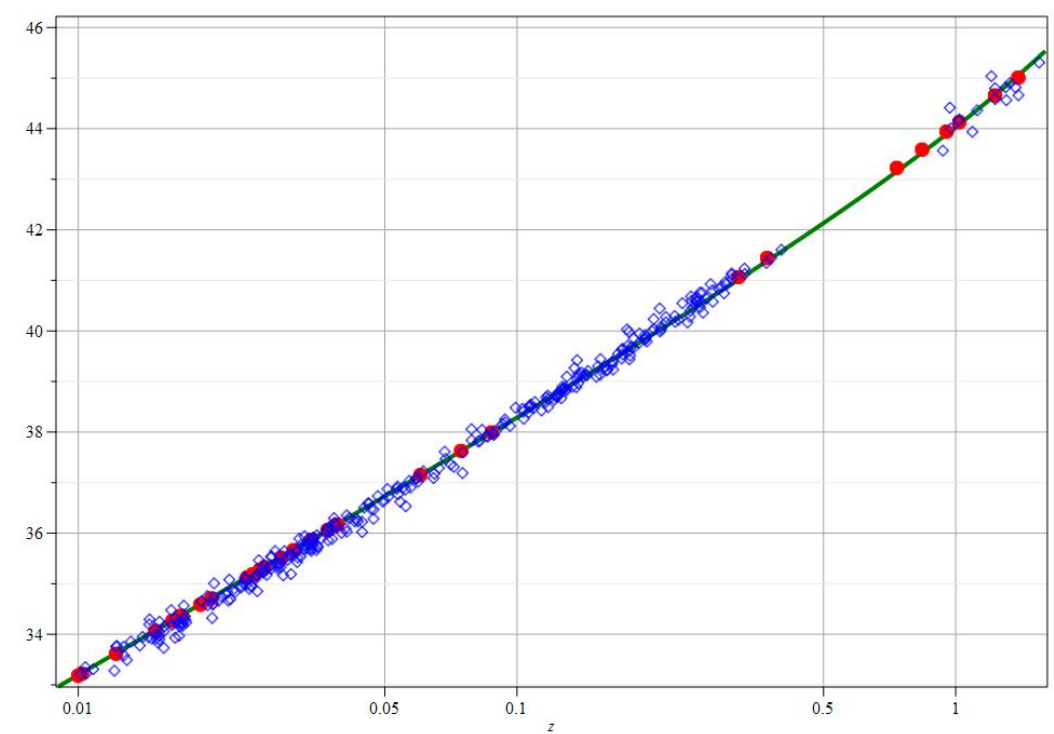

Fig. 3. The ratio "distance modulus - redshift" for type 1a supernovae in the usual (top graph) and logarithmic scales (bottom graph). Blue dots - 333 selected (with low distortion) data from a number of compilations and red dots - 33 "pure" supernovae outside or at the edge of galaxies [10]. Green line - theoretical curve $\mu-z$ in STC according to (59) at $\alpha=0.2$.

Fig. 3 shows a comparison of (59) with observational data on Type 1a supernovae (in the usual and logarithmic scales along $z$ axis), where $H_{0}=0.69 \mathrm{~h}$ is taken from the linear part and $\alpha=0.2$, which means that $\left(1+z_{E}\right)^{2}\left(1+z_{g}\right)^{-2} \sqrt{1+2 q_{0} z_{E}} \simeq\left(1+z_{D}\right)^{1-0.2 z}$.

It should be noted that this comparison with observations is preliminary and further clarification of its details is required, but even at this stage theory in the first approximation is consistent with observational data without additional hypotheses. The conclusion about a faster rate of time in the past is a direct consequence of general relativity for three-dimensional expanding space and observational data, while the assumption about the constancy of the rate of time was an artificial hypothesis.

\section{The early universe and the properties of $\mathrm{CMB}$}

\subsection{Early universe}

Let us consider the main modifications in the cosmology of the early epochs within the framework of STC. Let $a_{m r}=a\left(t_{r}\right)$ and $\rho_{m r}=\rho\left(t_{r}\right)$ correspond to the scale factor and density at $t_{r}$, corresponding to the end of the radiation-dominated epoch. Energy-momentum tensor of ultrarelativistic matter with density $\rho$ and pressure $p=\rho / 3$ :

$$
\begin{aligned}
& T^{i k}=\frac{d x^{i}}{d s} \frac{d x^{k}}{d s}(\rho+p)-p g^{i k}=\frac{4}{3} \rho \frac{d x^{i}}{d s} \frac{d x^{k}}{d s}-\frac{1}{3} \rho g^{i k}, \\
& T^{00}=\rho \frac{a^{2}}{a_{0}^{2} f}, \quad T_{0}^{0}=T^{00} g_{00}=\rho, \quad T_{00}=\rho g_{00}=\rho f \frac{a_{0}^{2}}{a^{2}},
\end{aligned}
$$

substituted into the energy-momentum conservation condition:

$$
\frac{1}{\sqrt{-g}} \frac{\partial}{\partial x^{0}}\left(T_{0}^{0} \sqrt{-g}\right)-\frac{1}{2} \frac{\partial g_{k l}}{\partial x^{0}} T^{k l}=0
$$


Leads to the standard relationships ( $\dot{a}=d a / d t)[6]$ :

$$
\frac{1}{a^{2}} \frac{\partial}{\partial t}\left(\rho a^{2}\right)+2 \rho \frac{\dot{a}}{a}=0, \quad \frac{\partial}{\partial t}\left(\rho a^{4}\right)=0, \quad \rho a^{4}=\rho_{m r} a_{m r}^{4} .
$$

The evolution equation (31) then turns into:

$$
\begin{gathered}
\frac{a^{2}}{a_{0}^{2} f} \frac{\dot{a}^{2}}{c^{2}}+k=\frac{8 \pi G \rho a^{4}}{3 c^{4}} \frac{1}{a^{2}}=\frac{a_{m r}^{2}}{a^{2}}, \quad a_{m r}^{2}=\frac{8 \pi G \rho a^{4}}{3 c^{4}}, \\
\dot{a}^{2}=c^{2} \frac{a_{0}^{2} f}{a^{2}}\left(\frac{a_{m r}^{2}}{a^{2}}-k\right), \quad \dot{a}= \pm c \frac{a_{0} \sqrt{f}}{a} \sqrt{\frac{a_{m r}^{2}}{a^{2}}-k} .
\end{gathered}
$$

Comparison with the Friedman equation for this case:

$$
\left(\frac{d a}{d \tau}\right)^{2}=c^{2}\left(\frac{a_{m r}^{2}}{a^{2}}-k\right), \frac{d a}{d \tau}= \pm c \sqrt{\frac{a_{m r}^{2}}{a^{2}}-k},
$$

shows that the expansion rate here also contains the factor $a_{0} / a$ leading to a faster expansion rate in early epochs in terms of the time of our epoch, as well as $\sqrt{f}$ associated with gravitational time dilation.

For proper time $\tau$ the standard expressions take place:

$$
c \tau=\int_{0}^{a(\tau)} \frac{a d a}{\sqrt{a_{m} a_{0}-k a^{2}}}= \begin{cases}\sqrt{a_{m r}^{2}}\left(1-\sqrt{1-a^{2} / a_{m r}^{2}}\right), & k=1 \\ \frac{a^{2}}{2 \sqrt{a_{m r}^{2}}}, & k=0 \\ \sqrt{a_{m r}^{2}}\left(\sqrt{1+a^{2} / a_{m r}^{2}}-1\right), & k=-1\end{cases}
$$

For the time $t \leq t_{r}$ with corresponding $a(t)$, the evolution equation (64) gives

$$
c t=\frac{1}{a_{0} \sqrt{f}} \int_{0}^{a(t)} \frac{a^{2} d a}{\sqrt{a_{m r}^{2}-k a^{2}}}= \begin{cases}\frac{a_{m}}{2 \sqrt{f}} \arcsin \left(\frac{a}{\sqrt{a_{m r}^{2}}}\right)-\frac{a}{2 a_{0} \sqrt{f}} \sqrt{a_{m r}^{2}-a^{2}}, & k=1, \\ \frac{a^{3}}{3 \sqrt{a_{m r}^{2} a_{0} f}}, & k=0, \\ \frac{a}{2 a_{0} \sqrt{f}} \sqrt{a_{m r}^{2}+a^{2}}-\frac{a_{m}}{2 \sqrt{f}} \operatorname{arcsh}\left(\frac{a}{\sqrt{a_{m r}^{2}}}\right), & k=-1\end{cases}
$$

\subsection{New treatment of the CMB properties}

The observed CMB, which arose in the very early universe, makes it possible to study closer epochs, in particular, the period of recombination, when radiation was disconnected from matter. Here we note some changes in the description of CMB, which follow from the main differences between STC and the previous models.

The faster rate of proper times in the early universe explains a number of facts for which radical hypotheses had to be advanced in the former treatments. In particular, if the scale factor was $10^{3}$ times smaller, then the light velocity, taking into account the gravitational time dilation, was at least an order of magnitude faster in terms of our time $\tilde{c} \sim 10 c$, which explains the homogeneity and isotropy of $\mathrm{CMB}$ by a much faster mixing of the photon flux, which practically eliminates the horizon problem. Moreover, since in the very early universe the 
particles of matter were ultrarelativistic, then they also moved at least an order faster than photons in our epoch, which explains the large-scale homogeneity and isotropy of all matter.

The ratio of the CMB temperature at an earlier epoch $T_{e}$ to its observed temperature $T_{r}$ is determined by the ratio of the observed wavelength $\lambda_{r}$ to the wavelength $\lambda_{e}$ at that epoch. In the Friedmann model, the redshift was attributed to the stretching of wavelengths and therefore the ratio of wavelengths was given by the ratio of scale factors $a_{0} / a$, i.e. depended on $z_{E}$ what gave for $T(a)$ :

$$
\frac{T_{e}}{T_{r}}=\frac{\lambda_{r}}{\lambda_{e}}=\frac{a_{0}}{a}=1+z_{E}, \quad \frac{d T}{T}=-\frac{d a}{a} .
$$

In STC, all redshifts of radiation are determined by the Doppler effect and GRC, as a result of which the temperature ratio now depends on $v(a)$ and $z_{g}$ :

$$
\frac{T_{e}}{T_{r}}=\frac{\lambda_{r}}{\lambda_{e}}=1+z_{g D}=\left(1+z_{g}\right) \sqrt{\frac{1+v / c}{1-v / c}} .
$$

As a result, the relationship between redshifts $z_{g D}$ and $a$ is now more complicated.

But the very fact that it appears $z_{g D}$ instead of $z_{E}$ already allows us to recognize a number of significant changes. Unlike linear stretching of wavelengths, the Doppler effect and GRC have a quadratic dependence on distance, which dominates for signals from the early universe, where they are of the same order. This means that CMB is almost completely described by this part of the effect.

For the same $a$, we have $z_{g D} \gg z_{E}$ and therefore re-interpretation of the observed redshift as a combination of GRC and Doppler shifts $z_{E} \rightarrow z_{g D}$ without changing its value means that it now refers to a larger $a$ and later time $t$ than previously thought. At $v \simeq c-\delta v$, $\delta v \ll c,(69)$ takes the form:

$$
\frac{T_{e}}{T_{r}}=\frac{\lambda_{r}}{\lambda_{e}} \simeq z_{g} z_{D} \simeq z_{g} \sqrt{\frac{2 c}{\delta v}} \simeq z_{g D} \simeq 1500 .
$$

so that the inhomogeneities in the recombination epoch, through which the relict radiation flow passed, would look flattened due to the relativistic contraction.

To estimate the order of magnitude, we will further assume $z_{g} \simeq z_{D}$ and, respectively $z_{g D} \simeq z_{g} z_{D} \simeq z_{D}^{2}$. Then (70) takes the form:

$$
\frac{T_{e}}{T_{r}}=\frac{\lambda_{r}}{\lambda_{e}} \simeq z_{D}^{2} \simeq \frac{2 c}{\delta v} \simeq 1500, \quad \frac{\delta v}{c} \simeq 0.0013 .
$$

The radial contraction ratio of the layer corresponding to the velocity $v=c-\delta v \simeq 0.9987 c$ is then of the order of 20. Thus, the layers of the recombination epoch are compressed due to the receding velocity by a factor of about 20 and approximately the same due to gravitational contraction, and the overall coefficient is of the order of 400 .

And, finally, one more change is associated with the measurement of the characteristics of $\mathrm{CMB}$ in our time, from which the value:

$$
\Omega_{m} h_{0}^{3} \simeq 0.096
$$

is determined [11]. From this value, assuming $\Omega_{m} \simeq 0.315$, then a value $h \simeq 0.67$ was fixed that significantly differed from $h \simeq 0.73$ determined from the redshifts and the apparent luminosities of Type 1a supernovae. On the contrary, in STC, the redshifts of supernovae fix the value $h \simeq 0.70$ (or $h \simeq 0.73$ ), and then $\Omega_{m}$ can be determined from the empirical relation for CMB 
(72), which gives $\Omega_{m} \simeq 0.28$ (or $\Omega_{m} \simeq 0.25$ ). These lower values are confirmed by other independent observations of the distribution of galaxies that give $\Omega_{m} \simeq 0.26$. Thus, in STC there is no contradiction between the data on supernovae and CMB; on the contrary, they only complement each other.

\section{Conclusion}

The standard model of relativistic cosmology, even with the dominance of hypothetical dark energy, leads to the doubling of redshifts due to the need to take into account in general relativity both the Doppler effect (due to the receding of the source in the observer's rest frame) and the stretching of the wavelengths during propagation of photons (due to the expansion of space). Since only a single redshift is observed, there is the double redshift problem. This problem means a catastrophic $(100 \%)$ discrepancy between theory and observations and the inconsistency of the former standard model of relativistic cosmology.

The fact is that in models with the Friedmann metric, to which the standard model of cosmology belongs, even in expanding space, the hypothesis of a static model about a constant rate of proper times during the evolution of the universe was preserved. However, in relativistic theories, the spacetime geometry is variable and usually the variability of the space geometry also leads to the variability of the rate of time. The hypothesis of the constancy of the time component of the metric is a very strong constraint that brings such models closer to Newtonian cosmology, where this constraint is natural.

STC model formulated in [6] with slowing down local proper times naturally solves the double redshift problem. In STC in earlier epochs, photons were emitted with a violetshift, which was compensated for during their propagation by the stretching redshift. As a result, at receiving the wavelengths contain only the contribution of the relativistic Doppler effect and GRC.

But where there is the Doppler effect, there is also an aberration, which leads to additional dimming of sources due to a decrease in the number of photons per unit solid angle. Therefore, STC differs from the Friedmann model also by taking into account the aberration.

The new "distance modulus - redshift" relation, following from STC, in the first approximation is consistent with the data on type 1a supernovae. STC leads to a number of nontrivial consequences for the early universe and allows to naturally solve the cosmological problems of previous models. The more complex dependence of the redshifts on the velocity and the scale factor makes it possible to reconcile the value $H_{0}$ from the data on the CMB and supernovae.

\section{Appendix. Relativistic aberration in cosmology}

Aberration is a change in direction towards a light source and its apparent luminosity due to its motion relative to the observer. The aberration of the light flux leads to the fact that the receding sources will appear dimmer than those at rest in relation to us at the same distance. But in the Friedmann model, in which redshifts were attributed to stretching, aberration was ignored for the same reason as the Doppler shift.

In relativistic kinematics, the Doppler effect and aberration are determined by the same factors, and their only difference is that the first effect expresses changes in the frequency and wavelength of a photon, and the second - in the intensity and direction of the light flux. Therefore, the presence of the Doppler effect also leads to an aberration of the light flux, and since the Doppler effect takes place in the STC, there must also be an aberration [6].

The solid angle element $d \varphi d\left(\cos \theta^{\prime}\right)$ in the source's rest frame is related to the angle $d \varphi d(\cos \theta)$ in the observer's rest frame by the Lorentz transformation for $\cos \theta^{\prime}$ :

$$
d(\cos \theta)=\frac{1-v^{2}}{\left(1+v \cos \theta^{\prime}\right)^{2}} d\left(\cos \theta^{\prime}\right) .
$$


For a flux from a receding source in a static frame in which the observer is at rest, $v \cos \theta^{\prime} \simeq-v$ and we find the observed solid angle element $\theta$ from:

$$
d(\cos \theta)=\frac{1+v}{1-v} d\left(\cos \theta^{\prime}\right)=\left(1+z_{D}\right)^{2} d\left(\cos \theta^{\prime}\right),
$$

where $\left(1+z_{D}\right)$ is the redshift factor for the longitudinal Doppler effect.

Thus, the change in the solid angle depends on the factor that determines the Doppler effect and these two effects will contribute together - the first decreases the frequency of photons, and the second - the number of photons in a given solid angle. With an increase in the solid angle of the flux cone, the number of photons in the cone of the same magnitude will decrease, and the apparent luminosity will decrease. This decrease in the density of the light flux from receding objects must be taken into account in the expanding universe.

Notice, that in the expanding space the solid angles in the light fluxes do not change. However, in our rest frame, the Doppler effect takes place already at the emission of the light flux, and therefore there will initially be an aberration, i.e. distortion of the solid angle of the flux. Further, this flux spreads in the expanding space without changing the solid angle.

The apparent luminosity is inversely proportional to the solid angle of the initial flux and is defined as $l=L / 4 \pi d_{p}^{2}$, where $d_{p}$ is the photometric distance, and $L$ is the absolute luminosity. Therefore, when aberration is taken into account, the apparent luminosities of distant objects $\tilde{l}$ will be lower $l$. This leads to an effective increase in the photometric distance $\tilde{d}_{p}$ with respect to $d_{p}$, in which the aberration is not taken into account:

$$
\tilde{l}=\frac{l}{\left(1+z_{D}\right)^{2}}=\frac{L}{4 \pi \tilde{d}_{p}^{2}}, \quad \tilde{d}_{p}=\left(1+z_{D}\right) d_{p} .
$$

Substituting the definition $d_{p}=\left(1+z_{D}\right) r_{k, \chi}$, where $r_{k, \chi}$ is the curvature radius of the sphere onto which the radiation spread, for the photometric distance, taking into account the aberration, we obtain:

$$
\tilde{d}_{p}=\left(1+z_{D}\right)^{2} r_{k, \chi}
$$

\section{References}

1.Tolman R. C. (1934) Relativity Therm. and Cosmology, O.U.P.

2.Einstein A. (1917) Sitz. Preuss. Akad. Wiss., 142.

3. Friedmann A. (1922). Z. Phys., 10, 377.

4. Zeldovich Y. B., Novikov I. D. (1983) Rel. Astroph. v.2 Struct. \& Evol. Univ., U.Ch.P.

5. Weinberg S. (2008) Cosmology, O.U.P.

6. Zakir Z. (2020) Quant. \& Grav. Phys., 1:008-7160.

7. Zakir Z. (2021) Quant. \& Grav. Phys., 2:011-7528.

8. Bondi H. (1947) MNRAS, 107, 410.

9. Mattig W. (1957) Astron. Nachr., 284, 109.

10. Pruzhinskaya M.V. et al. (2020) arXiv:2006.09433 astro-ph; (2011) Astron. Lett., 37, 663.

11. Planck 2018 results. VI. Cosmological parameters. arXiv:1807.06209. 